\title{
Approaches for Increasing the $\beta$-phase Concentration of Electrospun Polyvinylidene Fluoride (PVDF) Nanofibers
}

\author{
Guangshuai $\mathrm{Han}^{1}$, Yenfang $\mathrm{Su}^{1}$, Yining Feng ${ }^{1}$ and $\mathrm{Na} \mathrm{Lu}^{1,2,3^{*}}$
}

The electrospun PVDF has gained much attentions due to its excellent piezoelectricity, high flexibility, and low-cost. This study aims to develop the electrospun PVDF nanofibers with high $\beta$-phase concentration and piezoelectricity. The samples were prepared by the PVDF solution with different molecular weights. The earth abundant Zinc Oxide nanoparticles serve as the inorganic dopant to increase the polarization of the PVDF film during manufacturing process. The materials characterization methods, including Scanning Electron Microscopy, Energy Dispersive X-Ray, and Fourier-transform infrared spectroscopy were utilized to identify the material properties. The results indicate that the high molecular weight PVDF is favorable for electrospinning to obtain the high quality nanofibers. Furthermore, doping Zinc Oxide nanoparticles can effectively promote the polarization of electrospun PVDF nanofibers.

Keywords: PVDF; ZnO; Nanofiber; Electrospinning; Diameter distribution; Piezoelectric

Received 9 December 2019, Accepted 18 December 2019

DOI: $10.30919 / \mathrm{esmm} 5 \mathrm{f} 612$

\section{Introduction}

Piezoelectric materials have been widely used as actuators, sensors, transducers, ${ }^{1-4}$ and also energy harvester ${ }^{5-7}$ due to their unique mechanical-electrical coupling properties. It was first discovered by Pierre Curie in 1880. In principle, as mechanical stress is applied to a piezoelectric material, an electrical charge appears, and vice versa due to electromechanical coupling effect. There are various types of piezoelectric materials, including ceramics, polymers, even biological matter. Among the piezoelectric materials, piezoelectric polymers have gained more attentions due to high flexibility and the potential wide range applications.

Polyvinylidene fluoride (PVDF) is the polymer-based piezoelectric materials which have attracted much interests for energy harvesting applications due to the elastic properties. It can be applied in various fields such as aerospace, civil infrastructure, and biomedical engineering, etc. The molecular structure of PVDF consists of a repetition of a monomer vinylidene fluoride unit $\left(-\mathrm{CH}_{2} \mathrm{CF}_{2}-\right)_{\mathrm{n}}$. Among four semicrystalline polymorphs of PVFD ( $\alpha, \beta, \gamma$, and $\delta$ crystalline phases), the highly polarized $\beta$-phase of PVDF has desired electroactive properties, which is favorable for energy harvesting and sensing. The polymer chains of the $\beta$-phase (in the unit cell) are arranged in a way that all the dipoles are parallel between each other, leading to a net dipole moment. Therefore, the studies on $\beta$-phase enhancement in PVDF nanofibers is of vital importance for piezoelectric applications.

\footnotetext{
${ }^{\prime}$ Lyles School of Civil Engineering, Sustainable Materials and Renewable Technology (SMART) Lab, Purdue University, USA

${ }^{2}$ School of Materials Engineering, Purdue University, USA

${ }^{3}$ Center for intelligent infrastructure, Purdue University, USA

*E-mail: luna@purdue.edu
}

The post-processes, such as mechanical stretching and corona poling, are the comment treatment to improve the $\beta$-phase content of PVDF. The stretching and corona poling process proposed by $\mathrm{T}$ Kaur et $a l^{8}$ have obtained the piezoelectric constant $\mathrm{d}_{31} \sim 60 \mathrm{pC} / \mathrm{N}$ at $30{ }^{\circ} \mathrm{C}$. A. Salimi et al. ${ }^{9}$ suggested that stretching process can significantly improve the piezoelectric properties due to the uniform dipole moment by the orient beta phase crystals. Sencadas et al. ${ }^{10}$ proposed using the hightemperature pressing method to eliminate the porosity of PVDF film. The results of crystallization show that this method can decrease the porosity and further improve the $\beta$-phase content of PVDF.

The electrospinning synthesis process, on the other hand, is a popular method for generating $\beta$-phase PVDF nanofibers without any post-treatment. ${ }^{5,11-15}$ Instead of the post mechanical stretching and electrical poling, the dipoles spontaneously aligned in the electrospun nanofibers through the strong applied voltage and stretching force of the electrospinning process. The randomly oriented dipoles of $\alpha$-phase will transform into $\beta$-phase which is favorable for piezoelectric materials. Zhao et al. ${ }^{11}$ compared the properties of electrospun PVDF with various acetone amount and DMF ratio. It has found that polymer concentration and $\mathrm{DMF} /$ acetone ratio were the main parameters that affected the morphology of PVDF film. Zheng et al. ${ }^{16}$ discussed the polymorphism control of electrospun PVDF. Through adjusted the parameters such as decreasing electrospinning temperature, lower the feeding rate, and shorten the tip-to-collector distance, the $\beta$-phase of PVDF can be enhanced. Gafari et al..$^{15}$ developed a model from the experiments to predict the surface morphology and properties of PVDF nanofibers. They found that the parameters of the electrospinning process can be numerically optimized.

The PVDF film can be used as a nanogenerator for energy harvesting. The electrospun PVDF nanogenerators were found to have a high energy conversion efficiency of $5-2000 \mathrm{mV}^{5,6}$ and the electrical output depended on the applied force and frequency. The electrospun flexible PVDF thin film has shown the potential to harvest the 
mechanical force under high strain conditions.

Wang et al. ${ }^{12}$ fabricated the flexible electrospun PVDF force sensor with high sensitivity of $42 \mathrm{mV} / \mathrm{N}$. Ghafari and $\mathrm{Lu}^{4}$ synthesized the selfpolarized electrospun PVDF sensor. They found that the PVDF device can efficiently detect the transmitted acoustic wave at the frequency range from 1 to $100 \mathrm{kHz}$.

Besides, the oxide particles such as zine oxide $(\mathrm{ZnO})^{17-28}$ and graphene oxide ${ }^{29.35}$ are commonly used to improve the properties of nanomaterials including PVDF nanofibers. Among the oxides, $\mathrm{ZnO}$ has drawn great interest for decades owing to the versatility, environmentally friendly, earth abundancy, and excellent electrical properties. Moreover, $\mathrm{ZnO}$ has intrinsic wurtzite crystal structure which gives very good piezoelectric properties. Loh et al. ${ }^{25}$ investigated the performance of $\mathrm{ZnO}$ nanoparticle-polyelectrolyte thin film with different $\mathrm{ZnO}$ weight fractions. They suggested that $50 \%$ and $60 \% \mathrm{ZnO}$ polymer films display comparable piezoelectric properties without mechanical stretching and high-voltage poling. Ghafari et $a l^{23}$ explored the synthesis of $1 \mathrm{D} \mathrm{ZnO}$ nanofibers using the sol-gel approach and electrospinning process. A parametric study on the effects of annealing temperature, annealing time, and the effect of polymer concentration were conducted while maintaining the constant electrospinning parameters such as voltage and distance between syringe and collector plate. It was found that the higher polymer concentrations produced, the larger diameter $\mathrm{ZnO}$ fibers; whereas higher annealing temperatures produced lower diameter fibers. Di Mauro et $a l^{36}$ investigated the synthesis of $\mathrm{ZnO}$ nanofibers by electrospinning of a solution containing $\mathrm{ZnO}$ precursor, polyvinylpyrrolidone, and solvent. $\mathrm{He}$ further studied the effects of annealing temperatures and change in concentration on the electrospinning process and found that a change in concentration produced an effect on the morphology of the obtained $\mathrm{ZnO}$ nanofibers. Devi et al. $^{26}$ discussed the material properties of solvent casted PVDF-ZnO hybrid film. The results suggested that the $\mathrm{ZnO}$ has positive influence on electric and electronic properties of PVDF-ZnO composites. The stability of dispersion of particles was enhanced through surface grafting. Bafqi et al $^{27}$ electrospun the PVDF-ZnO fibers for characterization. It has found that the film with $15 \mathrm{wt} \% \mathrm{ZnO}$ can generate the output voltage up to $1.1 \mathrm{~V}$, which is potential for selfpowered wearable devices. Dodds et $a l^{28}$ enhanced the spin coated PVDF copolymer film with $\mathrm{ZnO}$ nanoparticles. The results indicated that the $\mathrm{ZnO}$ concentration has notable effects on remnant polarization of PVDF-ZnO film. The piezoelectric $\mathrm{ZnO}$ nanoparticles are indeed improved the piezoelectricity of PVDF.

Owing to the excellent flexibility and process simplicity of electrospun PVDF, this study is particularly interested in synthesis PVDF nanofibers via electrospinning. Built on the knowledge of previous literatures, it has known that the parameters of electrospinning procedure and solvent concentration affect the properties of PVDF film. However, none of the studies discussed the characters of electrospun PVDF synthesized from raw PVDF ingredients with different molecular weights. Moreover, the $\mathrm{ZnO}$ doped PVDF nanofibers are discussed. The morphology was observed through Scanning Electron Microscopy (SEM), and the material properties were characterized using Energy Dispersive X-Ray (EDX) and Fourier-transform infrared spectroscopy (FTIR). Finally, the relationship between the PVDF parameters and $\beta$ phase crystalline was discussed.

\section{Experiment program}

\subsection{Samples preparation}

The PVDF precursor solutions were prepared by mixing the solid-state PVDF with the organic solvent. The ratio of Dimethyl sulfoxide (DMSO, Sigma-Aldrich 99.5\%) and acetone (Sigma-Aldrich, 99.75\%) was 7:3 for the solvent. Two types of PVDF with different molecular weights were used, including PVDF pellets $\left(\mathrm{M}_{\mathrm{w}}=275,000\right.$, Sigma-Aldrich) and PVDF powder ( $\mathrm{M}_{\mathrm{w}}=534,000$, Sigma-Aldrich) for comparison. To obtain high $\beta$-phase concentration and enhanced the piezoelectricity, Zinc oxide nanoparticles $(\mathrm{ZnO}$, US research Nanomaterials. Inc., diameter from $10 \mathrm{~nm}$ to $30 \mathrm{~nm}$ ) was added into the PVDF precursors for electrospinning. Table 1 demonstrates the mix design for the $\mathrm{ZnO} / \mathrm{PVDF}$ nanofibers.

\subsection{Electrospinning process}

Electrospinning is a manufacturing method that can be used to synthesize nanofiber by applying electrostatic force to draw charged threads of polymer solutions. The electrospinning device consists of a high-voltage power source, syringe, needle and a grounded rotating collector. Fig. 1 shows the set-up of the electrospinning. The prepared solution is loaded into the syringe with a needle attached. The electrical potential is applied to the needle to eject the polymer at the desired flow

Table $1 \mathrm{ZnO/PVDF}$ nanofiber mix design.

\begin{tabular}{|c|c|c|c|}
\hline Sample & PVDF molecular weight & PVDF concentration & $\mathrm{ZnO}$ concentration \\
\hline HW & 534,000 & $14 \%$ & $0 \%$ \\
\hline $\mathrm{HW}-\mathrm{ZnO}$ & 534,000 & $14 \%$ & $1 \%$ \\
\hline LW & 275,000 & $14 \%$ & $0 \%$ \\
\hline $\mathrm{LW}-\mathrm{ZnO}$ & 275,000 & $14 \%$ & $1 \%$ \\
\hline
\end{tabular}

Table 2 Electrospinning parameters.

\begin{tabular}{cccc}
\hline Voltage & Distance between needle and collector & Flow rate & Needle \\
\hline $15 \mathrm{kV}$ & $10 \mathrm{~cm}$ & $2 \mathrm{ml} / \mathrm{h}$ & No. 21 \\
\hline
\end{tabular}


rate. Due to the applying of high voltage, the electrostatic force overcomes the surface tension of the polymer solution at the tip of the needle and forms a Taylor cone, which further elongates into a fluid jet. A rotating drum collects the fluid jetted fiber. The whipping motion of the polymer jet that appears between the needle and the collector allows the solvent to evaporate and remains the thin polymer film on the collector. The diameters of fibers range from the nano to the micron scale, depending on the processing parameters. Table 2 shows the parameters of the electrospinning process used by this study.

\subsection{Characterization of the samples}

FTIR (PerkinElmer Spectrum 100 FTIR) spectra were employed to determine the $\beta$-phase concentration of the samples by the Spectrometer. Through FTIR analysis, molecular structure and the chemical bonding of undetermined material will be provided. Previous literature has explicated the peak locations of $\alpha$ and $\beta$ phase PVDF on FTIR spectra. $^{37}$ The peak locations of $\alpha$ phase PVDF are shown at the wavelength of $531,614,766,796,870$, and $970 \mathrm{~cm}^{-1}$. On the other hand, the $\beta$ phase PVDF is presented at $440,470,510,840$, and 1280 $\mathrm{cm}^{-1}$. In this study, the sweeping wave numbers are range from $700 \mathrm{~cm}^{-1}$ to $1600 \mathrm{~cm}^{-1}$ since the wavenumber peaks of interests are $766 \mathrm{~cm}^{-1}(\alpha$ phase) and $840 \mathrm{~cm}^{-1}(\beta$ phase). The resolution for the FTIR measurement is $2 \mathrm{~cm}^{-1}$.

SEM (FEI Nove Nano-SEM) images were used to investigate the morphology of the nanofibers. The samples were sputter-coated by platinum. The obtained SEM images were further post-processed by the ImageJ software. EDX characterization was also used to identify the $\mathrm{ZnO}$ on the nanofibers.

\section{Results and discussion}

\subsection{FTIR results of PVDF nanofibers}

FTIR is an excellent tool to quantify the $\beta$-phase concentration of the PVDF nanofibers. According to the Lambert-Beer law, the relative concentration of the $\beta$-phase in a sample can be calculated by the equation below:

$$
F_{\beta}=\frac{A_{\beta}}{\left(K_{\beta} / K_{o}\right) A_{\alpha}+A_{\beta}}
$$

where $F_{\beta}$ is the calculated $\beta$-phase concentration; $A_{\alpha}$ and $A_{\beta}$ is the absorbance at $766 \mathrm{~cm}^{-1}$ and $840 \mathrm{~cm}^{-1} ; K_{\beta}$ and $K_{\alpha}$ is the absorption coefficients correspondingly.

Fig. 2 shows the FTIR spectra and the calculated the $\beta$-phase
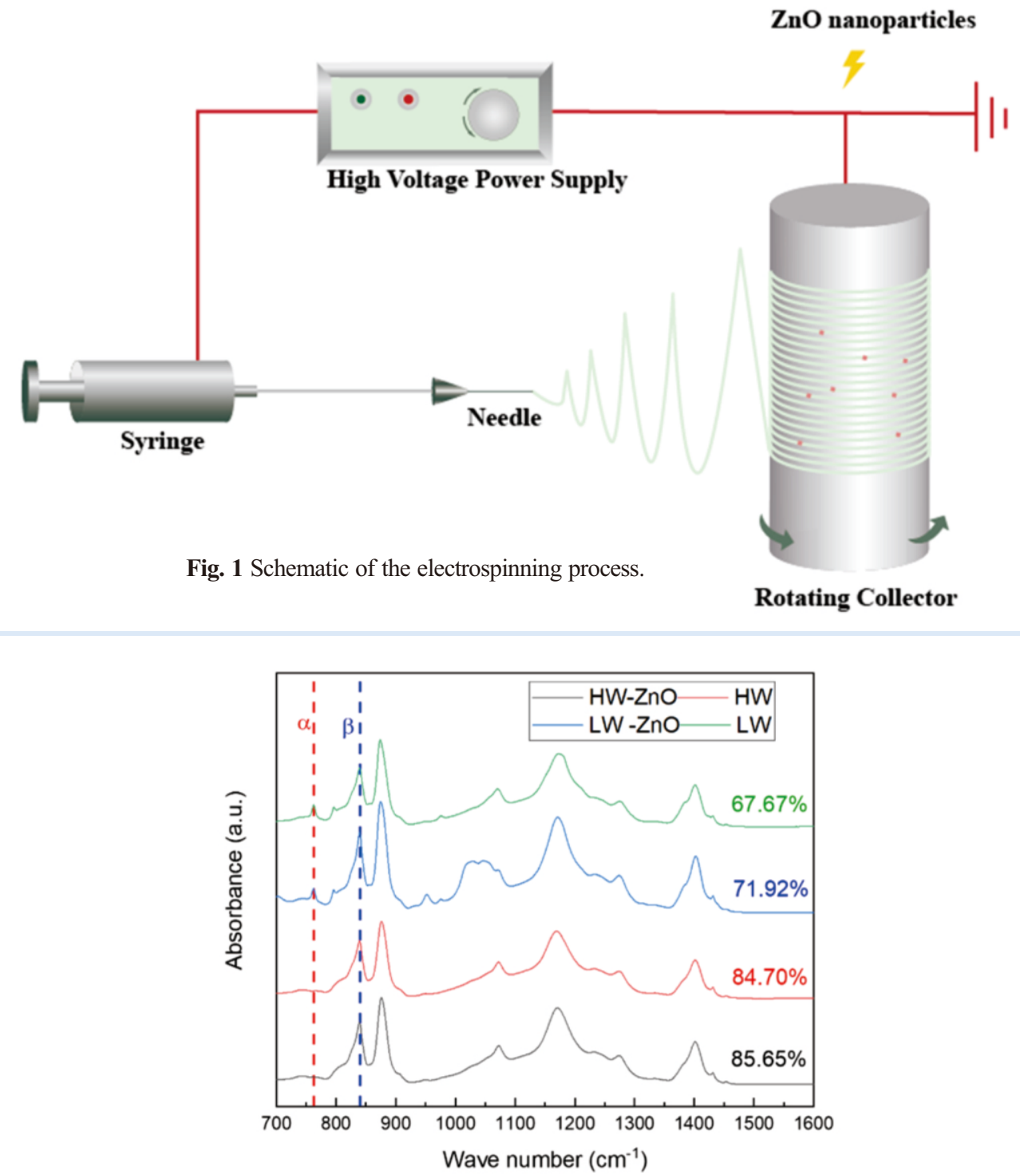

Fig. 2 FTIR spectrum and $\beta$-phase concentration for different PVDF nano-fiber. 
concentration of the prepared samples. The distinct peak at $840 \mathrm{~cm}^{-1}$ indicates that the electrospinning generates abundant $\beta$-phase PVDF. As for the PVDF nanofibers without $\mathrm{ZnO}$, the high molecular weight PVDF nanofibers have higher $\beta$-phase concentrations than the nanofibers prepared by the low molecular weight PVDF. The FTIR results also indicate that adding $\mathrm{ZnO}$ nanoparticles increases the $\beta$-phase concentration of the PVDF nanofibers. The following SEM images were used to discuss this in details.

\subsection{Morphology of PVDF nanofibers}

The PVDF nanofibers' diameters and beads formation are critical to the samples' performance. Fig. 3 demonstrates the morphology of the PVDF/ZnO nanofibers. Based on the EDX results shown in Fig. 3 (c) and (d), the $\mathrm{ZnO}$ nanoparticles uniformly distribute on the nanofibers. Therefore, preparing the uniform precursor solution mixed with $\mathrm{ZnO}$ is a feasible approach to dope $\mathrm{ZnO}$ nanoparticles with PVDF nanofibers. Fig. 3 (a) and (b) compare the PVDF nanofibers prepared by PVDF with different molecular weights. The SEM image (on the right) for the low molecular weight PVDF nanofibers displays plenty of beads. Conversely, there are no beads in the SEM image (on the left) for the high molecular weight PVDF. The different beads formation behavior has a good agreement with the FTIR results, which PVDF nanofibers prepared by the high molecular PVDF can generate more $\beta$-phase. The molecular weight of the PVDF affects the electrospinning process by changing the viscosity of the precursor solution. ${ }^{38}$ The product from high molecular PVDF usually has a denser structure and better mechanical performance. ${ }^{39,40}$ The high molecular PVDF powder is more suitable to perform the electrospinning process for high quality of PVDF nanofibers.

\subsection{Fiber distribution PVDF/ZnO nanofibers}

This section conducts the advanced analysis of the SEM images of the PVDF nanofibers. Fig. 4 compares the high molecular weight PVDF nanofibers with and without $\mathrm{ZnO}$. ImageJ software was utilized to perform the fiber diameter analysis. As shown in Fig. 4, before adding $\mathrm{ZnO}$ nanoparticles, the PVDF nanofibers' diameter mainly distribute in the range from $150 \mathrm{~nm}$ to $200 \mathrm{~nm}$. However, the fibers diameter of PVDF nanofibers with the $\mathrm{ZnO}$ nanoparticles tend to cluster in the range below $100 \mathrm{~nm}$. The reason is that the $\mathrm{ZnO}$ can help the precursor solution carry more electric charges. Thus, the electric field between the needle and rotating collector can apply more electrostatic force on the ejecting solution, which is preferable to stretch the fiber during the electrospinning process. The electrospun PVDF fibers then have more polarization at the same time. Therefore, adding $\mathrm{ZnO}$ nanoparticles can

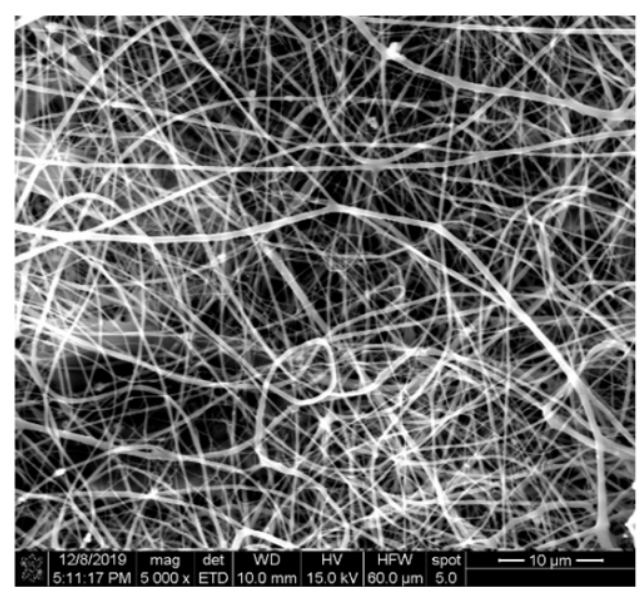

(a)

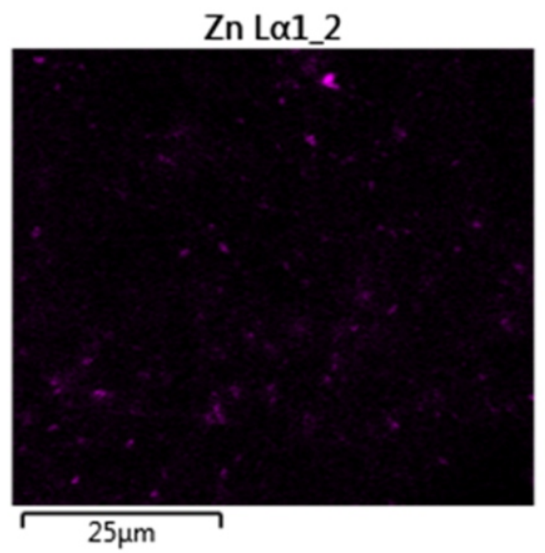

(c)

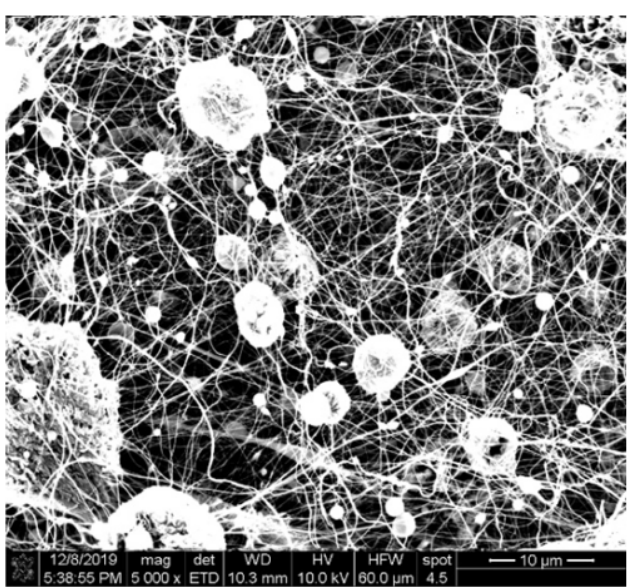

(b)

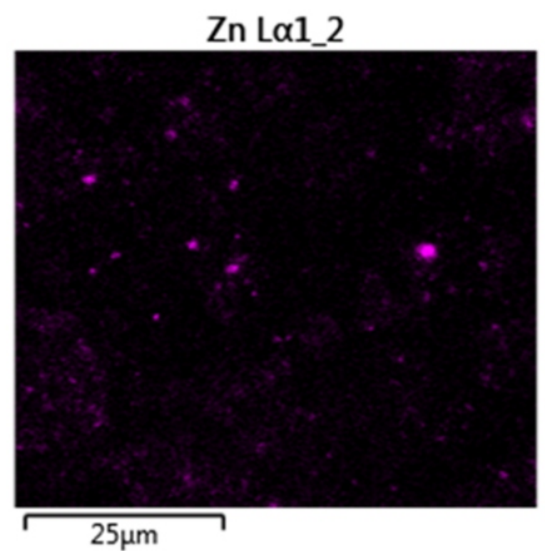

(d)

Fig. 3 (a) SEM images for high molecular weight PVDF/ZnO nanofibers (HW-ZnO); (b) SEM images for low molecular weight PVDF/ZnO nanofibers (LW-ZnO); (c) EDX Zinc atom distribution for high molecular weight PVDF/ZnO nanofibers (HW-ZnO); (d) EDX Zinc atom distribution for low molecular weight PVDF/ZnO nanofibers (LW-ZnO). 


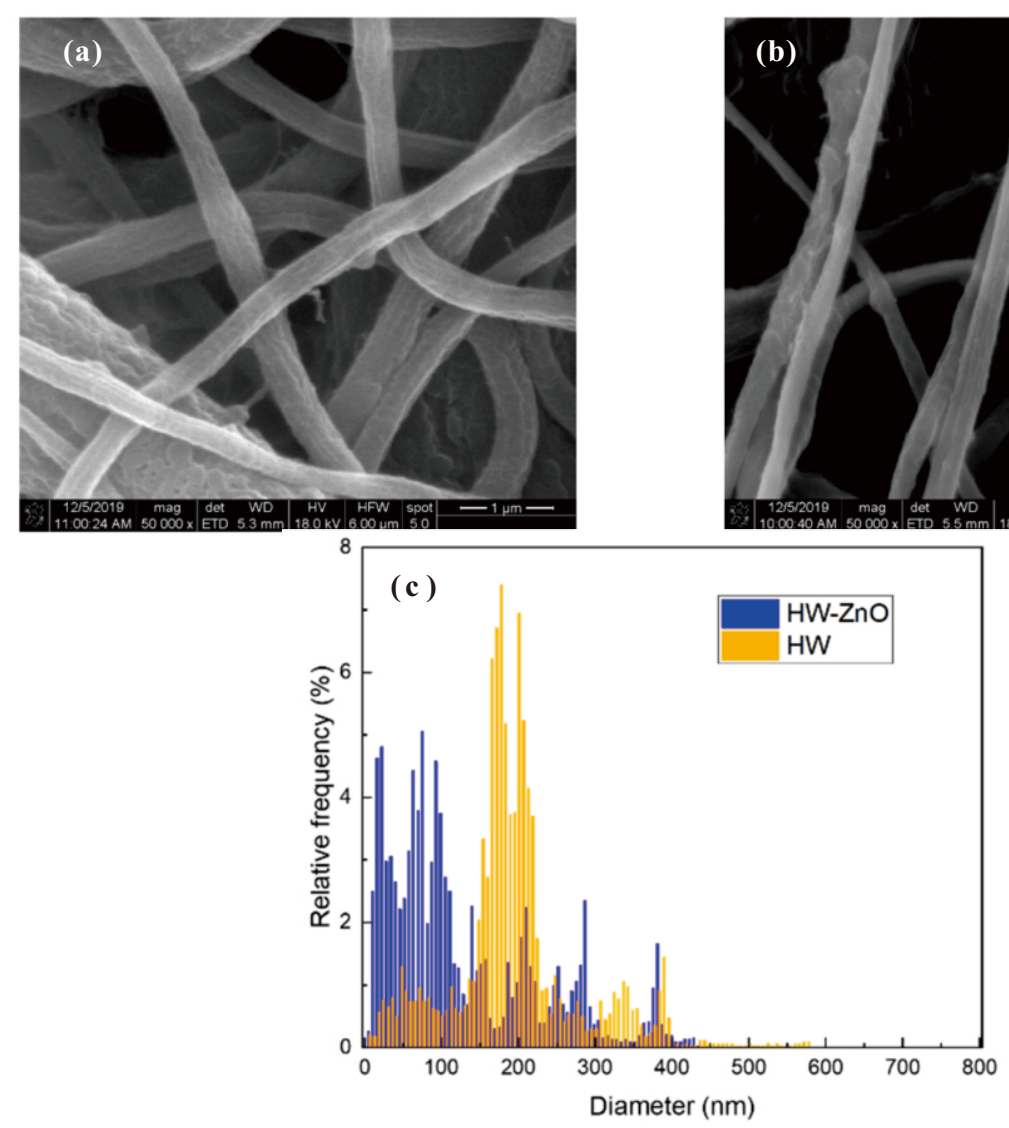

Fig. 4 (a) SEM images for high molecular weight PVDF nanofibers (HW); (b) SEM images for high molecular weight PVDF/ZnO nanofibers (HW$\mathrm{ZnO}$ ); (c) fiber diameter distribution comparison.
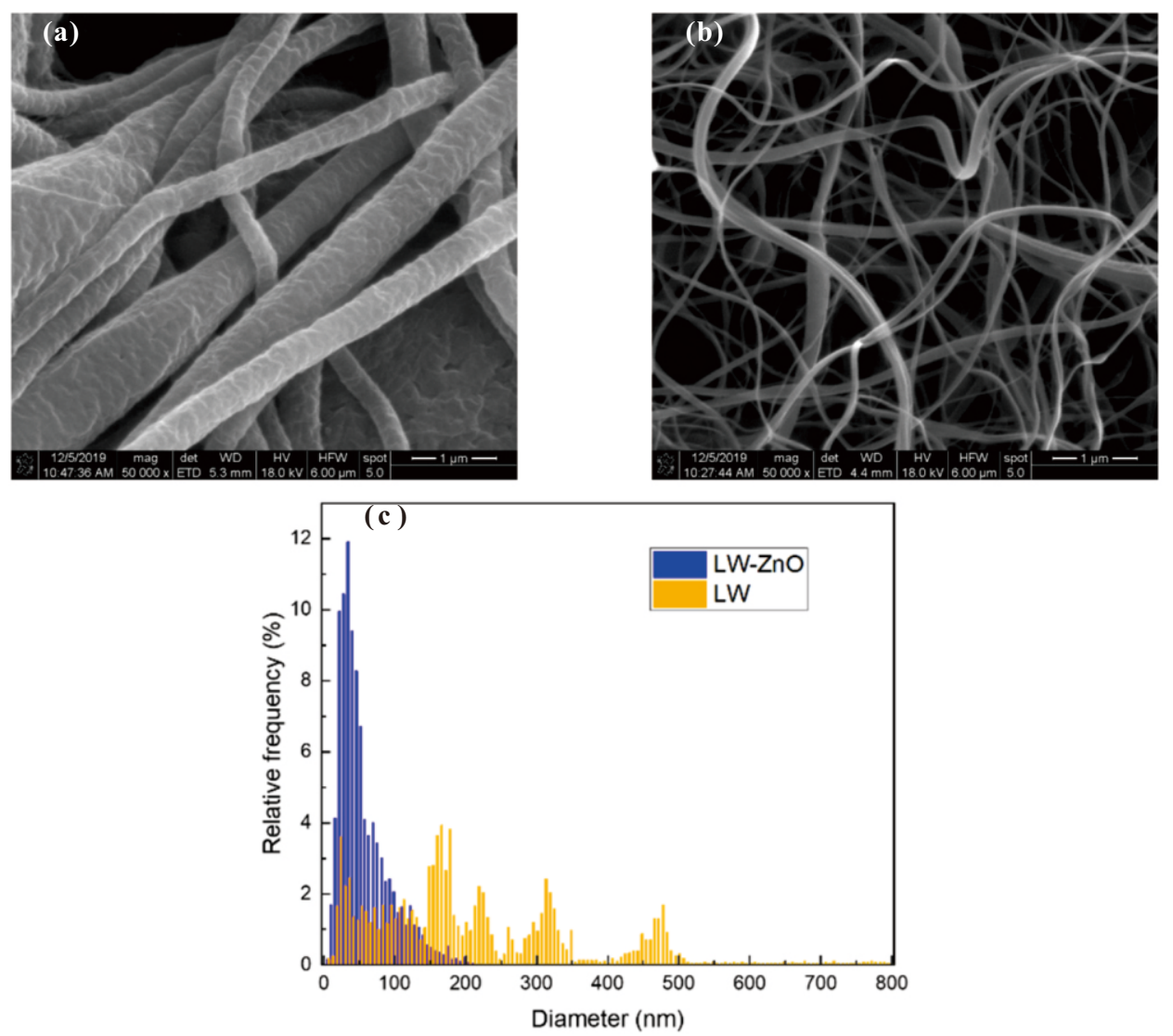

Fig. 5 (a) SEM images for low molecular weight PVDF nanofibers (LW); (b) SEM images for low molecular weight PVDF/ZnO nanofibers (LW-ZnO); (c) fiber diameter distribution comparison. 
increase the $\beta$-phase of the PVDF nanofibers.

Similarly, Fig. 5 compares the low molecular weight PVDF nanofibers with and without $\mathrm{ZnO}$ nanoparticles. A finer fiber distribution is observed when adding the $\mathrm{ZnO}$ nanoparticles in the PVDF nanofibers. This observation meets the abovementioned findings. The FTIR result has also verified the higher polarization of the PVDF/ZnO nanofibers.

\section{Conclusion}

A systematic study on enhancement of $\beta$-phase of electrospun PVDF nanofibers by different molecular weights of PVDF solution and addition of $\mathrm{ZnO}$ nanoparticle has been conducted and discussed. Through the obtained results, this study made a series of conclusion as follow. The electrospinning process can generate PVDF nanofibers with high $\beta$-phase concentration. Compare with the low molecular weight PVDF, the high molecular PVDF solution is favorable the electrospinning since the high molecular PVDF nanofibers have fewer beads. Incorporation of $\mathrm{ZnO}$ nanoparticles can effectively increase the polarization of the electrospun PVDF nanofibers by carrying more electric charges. This process also decreases the fibers' sizes and increases the $\beta$-phase concentration of the PVDF nanofibers. In conclusion, the combination of the high molecular weight PVDF powders and $\mathrm{ZnO}$ nanoparticles has the strong potential to fabricate the high-quality materials for the piezoelectric device.

\section{Acknowledgment}

The authors thank Purdue Research Foundation for their generous financial support.

\section{References}

1. Y. F. Su, G. Han, A. Amran, T. Nantung and N. Lu, Constr. Build. Mater, 2019, 225, 340-347.

2. E. Ghafari, Y. Yuan, C. Wu, T. Nantung and N. Lu, Constr. Build. Mater. 2018, 171, 504-510.

3. H. Gu, G. Song, H. Dhonde, Y. L. Mo and S. Yan, Smart Mater. Struct., 2006, 15, 1837-1845.

4. E. Ghafari and N. Lu, Compos. Part B-Eng., 2019, 160, 1-9.

5. C. Chang, V. H. Tran, J. Wang, Y. K. Fuh and L. Lin, Nano Lett., 2010, 10, 726-731.

6. J. Fang, X. Wang and T. Lin, J. Mater. Chem., 2011, 21, 11088.

7. Y. F. Su, R. R. Kotian and N. Lu, Compos. Part B-Eng., 2018, 153, 124-129.

8. T. Kaura, R. Nath and M. M. Perlman, J. Phys. D Appl. Phys., 1991, 24, 1848-1854

9. A. Salimi and A. A. Yousefi, J. Polym. Sci. Phys., 2004, 42, 8.

10. V. Sencadas, R. Gregorio Filho and S. Lanceros-Mendez, J. Non-Cryst. Solids, 2006, 352, 2226-2229.

11. Z. Zhao, J. Li, X. Yuan, X. Li, Y. Zhang and J. Sheng, J Appl. Polym. Sci., $2005,97,466-474$.

12. Y. R. Wang, J. M. Zheng, G. Y. Ren, P. H. Zhang and C. Xu, Smart Mater. Struct., 2011, 20, 045009

13. J. A. Epaarachchi, B. Li, A. K.T. Lau, J. Zheng, C. Xu and J. Leng, Opt.
Eng., 2013, 8793, 879314.

14. Y. Liao, R. Wang, M. Tian, C. Qiu and A. G. Fane, J. Membrane Sci., 2013 , 425-426, 30-39.

15. E. Ghafari, X. Jiang and N. Lu, Adv. Compos. Hybrid Mater., 2017, 1, 332340.

16. J. Zheng, A. He, J. Li and C. C. Han, Macromol. Rap. Commun., 2007, 28 , 2159-2162.

17. B. Kucukgok, B. Wang, A. G. Melton, N. Lu and I. T. Ferguson, Phys. Stat. Sol. C, 2014, 11, 894-897.

18. F. H. Teherani, D. C. Look, D. J. Rogers, B. Hussain, B. Kucukgok, M. Y. A. Raja, B. Klein, N. Lu and I. T. Ferguson, Opt. Eng., 2014, 8987, 898718.

19. E. Ghafari, S. A. Ghahari, Y. Feng, F. Severgnini and N. Lu, Compos. Part BEng., 2016, 105, 160-166.

20. B. Kucukgok, B. Hussain, C. Zhou, I. T. Ferguson and N. Lu, MRS Proceedings, 2015, 1805

21. S. Ghahari, E. Ghafari and N. Lu, Constr. Build. Mater, 2017, 146, 755-763.

22. F. H. Teherani, D. C. Look, D. J. Rogers, C. Zhou, A. Ghods, K. L. Yunghans, V. G. Saravade, P. V. Patel, X. Jiang, B. Kucukgok, N. Lu and I. Ferguson, Opt. Eng., 2017, 10105, 101051K.

23. E. Ghafari, Y. Feng, Y. Liu, I. Ferguson and N. Lu, Compos. Part B-Eng., 2017, 116, 40-45.

24. D. J. Rogers, D. C. Look, F. H. Teherani, V. G. Saravade, I. T. Ferguson, A. Ghods, A. M. Elquist, Y. Wang, C. Zhou and N. Lu, Opt. Eng., 2018, 10553, 10533R.

25. K. J. Loh and D. Chang, J. Mater. Sci., 2010, 46, 228-237.

26. P. I. Devi and K. Ramachandran, J. Exp. Nanosci., 2011, 6, 281-293.

27. M. S. Sorayani Bafqi, R. Bagherzadeh and M. Latifi, J. Polym. Res., 2015, 22, 130-139.

28. J. S. Dodds, F. N. Meyers and K. J. Loh, IEEE Sens. J., 2012, 12, 1889-1890.

29. W. Deng, T. Kang, H. Liu, J. Zhang, N. Wang, N. Lu, Y. Ma, A. Umar and Z. Guo, Sci. Adv. Mater., 2018, 10, 937-949.

30. M. Idrees, S. Batool, J. Kong, Q. Zhuang, H. Liu, Q. Shao, N. Lu, Y. Feng, E. K. Wujcik, Q. Gao, T. Ding, R. Wei and Z. Guo, Electrochim. Acta, 2019, 296, 925-937.

31. Z. Zhang, J. Zhang, S. Li, J. Liu, M. Dong, Y. Li, N. Lu, S. Lei, J. Tang, J. Fan and Z. Guo, Compos. Part B-Eng., 2019, 176, 107338.

32. X. Gong, Y. Liu, Y. Wang, Z. Xie, Q. Dong, M. Dong, H. Liu, Q. Shao, N. Lu, V. Murugadoss, T. Ding and Z. Guo, Polymer, 2019, 168, 131-137.

33. J. X. Zhang, Y. X. Liang, X. Wang, H. J. Zhou, S. Y. Li, J. Zhang, Y. Feng, N. Lu, Q. Wang and Z. Guo, Adv. Compos. Hybrid Mater, 2017, 1, 300-309.

34. M. Ataur Rahman and G. S. Chung, J. Alloys Compound., 2013, 581, 724 730.

35. M. Abbasipour, R. Khajavi, A. A. Yousefi, M. E. Yazdanshenas and F. Razaghian, J. Mater. Sci-Mater. El., 2017, 28, 15942-15952.

36. A. Di Mauro, M. Zimbone, M. E. Fragalà and G. Impellizzeri, Mat. Sci. Semicon. Proc., 2016, 42, 98-101.

37. F. Mokhtari, M. Latifi and M. Shamshirsaz, The Journal of The Textile Institute, 2015, 107, 1-19.

38. Z. Chen, D. Rana, T. Matsuura, D. Meng and C. Q. Lan, Chem. Eng. J., 2015, 276, 174-184.

39. N. Li, Z. Chang, Q. Lu, C. Xiao, J. Wu and C. Liang, Text. Res. J., 2018, 89, 1311-1320.

40. S. Rajabzadeh, D. Ogawa, Y. Ohmukai, Z. Zhou, T. Ishigami and H. Matsuyama, Desalin. Water Treat., 2015, 54, 2911-2919.

Publisher's Note Engineered Science Publisher remains neutral with regard to jurisdictional claims in published maps and institutional affiliations. 